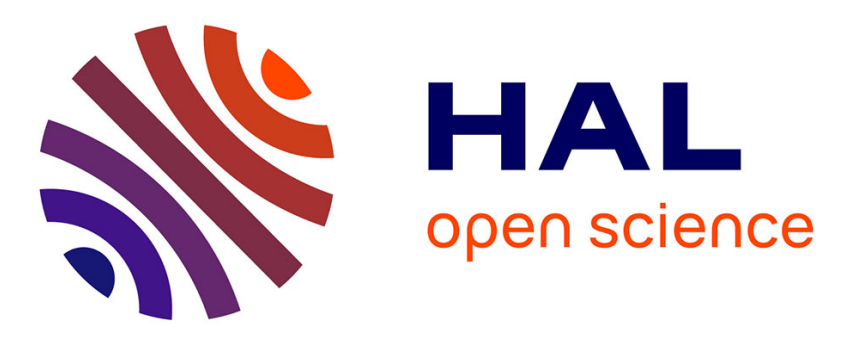

\title{
Etude du fonctionnement des photopiles solaires Cu2S-CdS type S.A.T.
}

\author{
H. Luquet, G.-M. Moussalli, J. Bougnot, M. Perotin, M. Savelli
}

\section{To cite this version:}

H. Luquet, G.-M. Moussalli, J. Bougnot, M. Perotin, M. Savelli. Etude du fonctionnement des photopiles solaires Cu2S-CdS type S.A.T.. Revue de Physique Appliquée, 1979, 14 (1), pp.245-251. 10.1051/rphysap:01979001401024500 . jpa-00244583

\section{HAL Id: jpa-00244583 https://hal.science/jpa-00244583}

Submitted on 1 Jan 1979

HAL is a multi-disciplinary open access archive for the deposit and dissemination of scientific research documents, whether they are published or not. The documents may come from teaching and research institutions in France or abroad, or from public or private research centers.
L'archive ouverte pluridisciplinaire HAL, est destinée au dépôt et à la diffusion de documents scientifiques de niveau recherche, publiés ou non, émanant des établissements d'enseignement et de recherche français ou étrangers, des laboratoires publics ou privés. 


\title{
Etude du fonctionnement des photopiles solaires $\mathrm{Cu}_{2} \mathrm{~S}-\mathrm{CdS}$ type S.A.T.
}

\author{
H. Luquet, G.-M. Moussalli, J. Bougnot, M. Perotin et M. Savelli \\ Centre d'Etudes d'Electronique des Solides $\left({ }^{*}\right)$, Université des Sciences et Techniques du Languedoc, \\ 34060 Montpellier Cedex, France
}

(Reçu le 28 juin 1978, révisé le 13 octobre 1978, accepté le 16 octobre 1978)

\begin{abstract}
Résumé. - Dans cet article nous montrons que dans les photopiles $\mathrm{Cu}_{2} \mathrm{~S}-\mathrm{CdS}$ (SAT) les lois de courants à l'obscurité et sous éclairement correspondent à des mécanismes, de conduction par effet tunnel. De plus en supposant l'existence d'une couche interfaciale limitée par des états d'interfaces entre le $\mathrm{Cu}_{2} \mathrm{~S}$ et le $\mathrm{CdS}$, nous avons pu expliquer les résultats concernant les mesures de la capacité en fonction de la tension appliquée.
\end{abstract}

\begin{abstract}
In this article we have shown that tunneling current laws in the dark and under illumination in SAT $\mathrm{Cu}_{2} \mathrm{~S}-\mathrm{CdS}$ photocells correspond to a tunnel conduction mechanism. In addition, assuming the existence of an interface layer limited by interface states between $\mathrm{Cu}_{2} \mathrm{~S}$ and $\mathrm{CdS}$, we have explained the results concerning capacitance measurements as a function of the applied voltage.
\end{abstract}

1. Introduction. - Dans cet article, nous présentons les résultats concernant le mode de conduction à l'obscurité et sous éclairement des cellules $\mathrm{Cu}_{2} \mathrm{~S}-\mathrm{CdS}$ fabriquées par la Société Anonyme des Télécommunications (SAT) [1-6]. Sur un support zingué, 40 à $45 \mu$ de CdS dopé ou non sont évaporés; ensuite par échange d'ions dans une solution de chlorure cuivreux, le $\mathrm{CdS}$ est transformé en $\mathrm{Cu}_{2} \mathrm{~S}$ sur une épaisseur de $1 \mu$ environ; une grille d'or assure le contact sur le $\mathrm{Cu}_{2} \mathrm{~S}$.

Nous avons étudié deux types de cellules :

Type $\mathbf{N}$ : cellules dites normales - CdS non dopé. Ces photopiles ont un courant de court-circuit $I_{\mathrm{cc}} \simeq 20 \mathrm{~mA} / \mathrm{cm}^{2}$, une tension en circuit ouvert $V_{\mathrm{co}} \simeq 450 \mathrm{mV}$ et un rendement proche de $6 \%$.

Type D I, D II : cellules dont le CdS a été dopé par introduction d'impuretés dans la poudre avant évaporation. Nous avons eu deux sortes de cellules de ce type correspondant à deux impuretés. Les caractéristiques sont les suivantes :

$I_{\text {cc }} 21 \mathrm{~mA} / \mathrm{cm}^{2}$ et $18 \mathrm{~mA} / \mathrm{cm}^{2}$ pour D II et D I $V_{\text {co }} 425 \mathrm{mV}$ et $435 \mathrm{mV}$ pour D II et D I

$\eta \quad 6 \%$ et $5,5 \%$ pour D II et D I.

2. Résultats expérimentaux. -2.1 CARACTÉRISTIQUES COURANT-TENSION. - 2.1.1 Polarisation directe. - Sur les figures 1 et 2 nous présentons pour deux

$\left(^{*}\right)$ Associé au C.N.R.S.

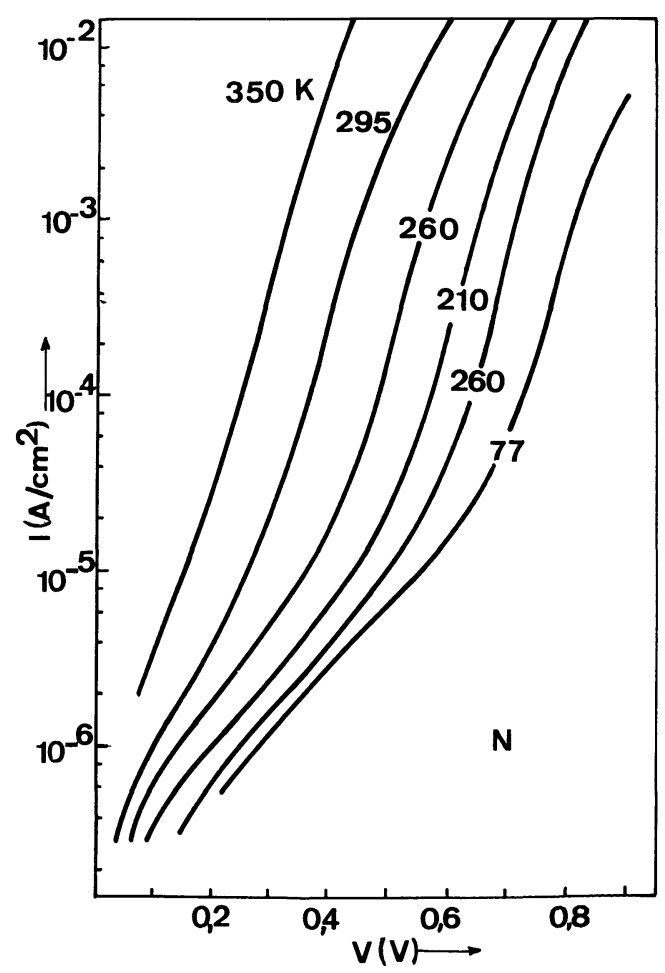

Fig. 1. - Evolution des caractéristiques directes $I-V$ en fonction de la température dans le cas d'une cellule de type $\mathrm{N}$.

types de cellules $\mathrm{N}$ et $\mathrm{D}$ I, la variation du courant direct avec la tension appliquée et pour différentes températures. Cette variation peut être représentée 


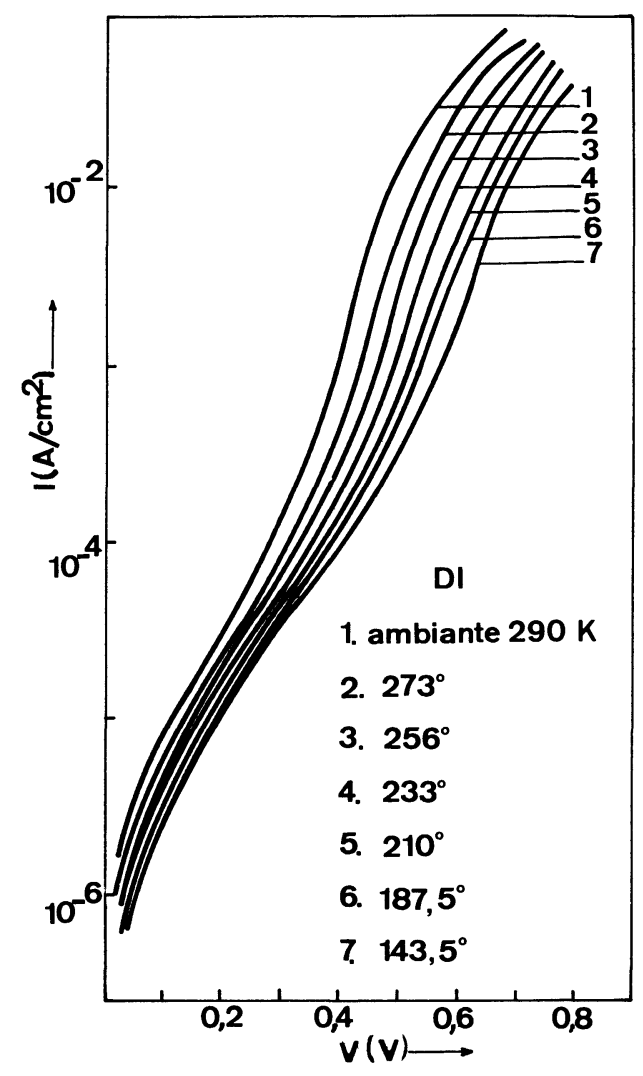

Fig. 2. - Evolution des caractéristiques directes $I$ - $V$ en fonction de la température dans le cas d'une cellule de type D I.

par la somme de deux courants $I_{1}$ et $I_{2}$ et se mettre sous la forme [8] :

$I=I_{1}+I_{2}=I_{\mathrm{S} 1}\left(\exp a_{1} V-1\right)+I_{\mathrm{S} 2} \exp a_{2} V$

avec

$I_{\mathrm{S} 1}>I_{\mathrm{S} 2}$ et $a_{1}<a_{2}$.

En fonction de la température, la loi de décomposition se conserve, les paramètres $a_{1}$ et $a_{2}$ variant peu avec la température. Les courants de saturation $I_{\mathrm{S} 1}$ et $I_{\mathrm{S} 2}$ suivent une loi empirique en fonction de la température de la forme :

$I_{\mathrm{S}}=I_{\mathrm{S} 0} \exp c T$.

(2) $I=I_{\mathrm{Br}} \exp k_{\mathrm{Br}} V$.
Dans le tableau I nous donnons pour N, D I et $\mathrm{D}$ II, les valeurs moyennes des différents paramètres : $I_{\mathrm{S} 1}, I_{\mathrm{S} 2}, a_{1}, a_{2}, c$.

Tableau I.

\begin{tabular}{|c|c|c|c|c|c|}
\hline \multicolumn{3}{|c|}{$T=300 \mathrm{~K}$} & \multicolumn{3}{|c|}{$100 \mathrm{~K} \leqslant T \leqslant 300 \mathrm{~K}$} \\
\hline & $\begin{array}{r}I_{\mathrm{S} 1} \mathrm{~A} / \mathrm{cm}^{2} \\
6,0 \times 10^{-7}\end{array}$ & $\begin{array}{c}a_{1}\left(\mathrm{~V}^{-1}\right) \\
11\end{array}$ & $\begin{array}{r}I_{\mathrm{SO}_{1}} \mathrm{~A} / \mathrm{cm}^{2} \\
4,3 \times 10^{-9}\end{array}$ & $\begin{array}{c}c_{1} \\
0,016\end{array}$ & $\begin{array}{c}a_{1} \\
10\end{array}$ \\
\hline $\mathbf{N}$ & $\begin{array}{c}I_{\mathrm{S2} 2} \mathrm{~A} / \mathrm{cm}^{2} \\
5,6 \times 10^{-10}\end{array}$ & $\begin{array}{c}a_{2}\left(\mathrm{~V}^{-1}\right) \\
35\end{array}$ & $\begin{array}{c}I_{\mathrm{SO}_{2}} \mathrm{~A} / \mathrm{cm}^{2} \\
7,3 \times 10^{-23}\end{array}$ & $\begin{array}{c}c_{2} \\
0,100\end{array}$ & $\begin{array}{c}a_{2} \\
36\end{array}$ \\
\hline & $\begin{array}{r}I_{\mathrm{S1}} \mathrm{A} / \mathrm{cm}^{2} \\
7,4 \times 10^{-6}\end{array}$ & $\begin{array}{c}a_{1}\left(\mathrm{~V}^{-1}\right) \\
9,0\end{array}$ & $\begin{array}{r}I_{\mathrm{SO}_{1}} \mathrm{~A} / \mathrm{cm}^{2} \\
1,4 \times 10^{-6}\end{array}$ & $\begin{array}{c}c_{1} \\
0,006\end{array}$ & $\begin{array}{l}a_{1} \\
9,3\end{array}$ \\
\hline DI & $\begin{array}{c}I_{\mathrm{S} 2} \mathrm{~A} / \mathrm{cm}^{2} \\
2 \times 10^{-9}\end{array}$ & $\begin{array}{c}a_{2}\left(\mathrm{~V}^{-1}\right) \\
30,6\end{array}$ & $\begin{array}{c}I_{\mathrm{SO}_{2}} \mathrm{~A} / \mathrm{cm}^{2} \\
3,5 \times 10^{-13}\end{array}$ & $\begin{array}{c}c_{2} \\
0,027\end{array}$ & $\begin{array}{c}a_{2} \\
30,8\end{array}$ \\
\hline & $\begin{array}{r}I_{\mathrm{S1} 1} \mathrm{~A} / \mathrm{cm}^{2} \\
6,3 \times 10^{-6}\end{array}$ & $\begin{array}{c}a_{1}\left(\mathrm{~V}^{-1}\right) \\
9,1\end{array}$ & $\begin{array}{r}I_{\mathrm{SO}_{1}} \mathrm{~A} / \mathrm{cm}^{2} \\
1,0 \times 10^{-7}\end{array}$ & $\begin{array}{c}c_{1} \\
0,007\end{array}$ & $\begin{array}{l}a_{1} \\
9\end{array}$ \\
\hline D & $\begin{array}{r}I_{\mathrm{S} 2} \mathrm{~A} / \mathrm{cm}^{2} \\
4,6 \times 10^{-9}\end{array}$ & $\begin{array}{c}a_{2}\left(\mathrm{~V}^{-1}\right) \\
31,7\end{array}$ & $\begin{array}{c}I_{\mathrm{SO}_{2}} \mathrm{~A} / \mathrm{cm}^{2} \\
3,5 \times 10^{-16}\end{array}$ & $\begin{array}{c}c_{2} \\
0,051\end{array}$ & $\begin{array}{c}a_{2} \\
32\end{array}$ \\
\hline
\end{tabular}

Sous éclairement, les cellules de la SAT ne présentent pas de recoupement important en polarisation directe entre la caractéristique à l'obscurité et celle sous éclairement. Le courant total de la cellule $(I)$ peut se décomposer en un courant dû à la polarisation $\left(I^{*}\right)$ et un courant dû à la création de porteurs par effet photovoltaïque $\left(I_{\mathrm{cc}}\right)$ soit $: I=I^{*}-I_{\mathrm{cc}}$. Le courant $I^{*}$ a la même forme [9] que le courant de la jonction à l'obscurité et peut être représenté par les équations (1) et (2). Dans le tableau II, pour une diode caractéristique du type $\mathrm{D}$ I nous montrons à titre d'exemple l'évolution des paramètres sous l'effet de l'éclairement.

Nous remarquons surtout une augmentation importante du courant de saturation $I_{\mathbf{S} 1}$ sous éclairement, ce qui est néfaste pour les performances de la cellule.

2.1.2 Polarisation inverse. - Au-dessus d'une tension $V_{\mathbf{B r}}$ nous observons pour la caractéristique courant-tension un changement de loi de conduction (Fig. 3). Dans le domaine compris entre la tension $V_{\mathrm{Br}}$ et la tension où se manifeste la résistance série, on peut traduire le courant par une loi du type

Tableau II

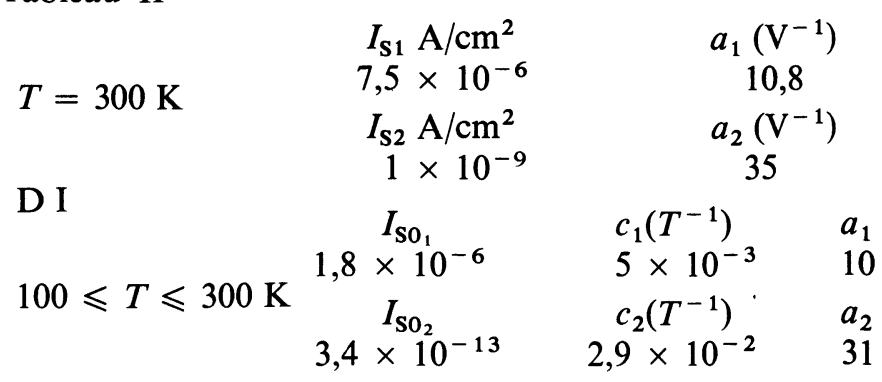

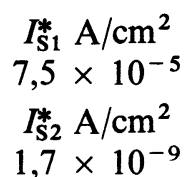

$I_{\mathrm{SO}}^{*}$

$3,9 \times 10^{-5}$

$I_{\mathrm{SO}_{2}}^{*}$

$2,1 \times 10^{-14}$

$$
\begin{gathered}
a_{1}^{*}\left(\mathrm{~V}^{-1}\right) \\
9,8 \\
a_{2}^{*}\left(\mathrm{~V}^{-1}\right) \\
36,8
\end{gathered}
$$

$\begin{array}{cc}c_{1}^{*}\left(T^{-1}\right) & a_{1}^{*} \\ 2 \times 10^{-3} & 8,3 \\ c_{2}^{*}\left(T^{-1}\right) & a_{2}^{*} \\ 3,6 \times 10^{-2} & 36,4\end{array}$



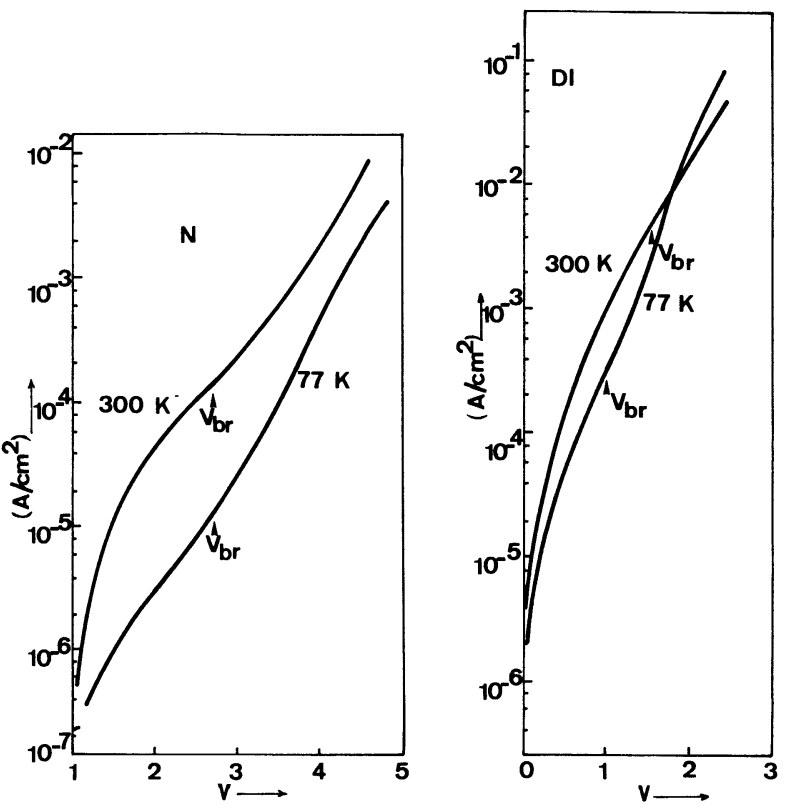

Fig. 3. - Caractéristiques inverses pour deux types de cellules à $300 \mathrm{~K}$ et à $77 \mathrm{~K}$.

Dans le tableau III nous donnons les valeurs moyennes des paramètres $V_{\mathrm{Br}}, I_{\mathrm{Br}}$ et $k_{\mathrm{Br}}$ pour les cellules $\mathrm{N}, \mathrm{D}$ I et D II.

Tableau III.

\begin{tabular}{|c|c|c|c|c|}
\hline & & $\begin{array}{c}V_{\mathrm{Br}}(\mathrm{V}) \\
-\end{array}$ & $I_{\mathrm{Br}} \mathrm{A} / \mathrm{cm}^{2}$ & $k_{\mathrm{Br}}\left(\mathrm{V}^{-1}\right)$ \\
\hline $\mathbf{N}$ & $\begin{array}{r}300 \mathrm{~K} \\
77 \mathrm{~K}\end{array}$ & $\begin{array}{l}2,75 \\
2,60\end{array}$ & $\begin{array}{l}1,62 \times 10^{-7} \\
4,83 \times 10^{-8}\end{array}$ & $\begin{array}{l}2,56 \\
2,56\end{array}$ \\
\hline D I & $\begin{array}{r}300 \mathrm{~K} \\
77 \mathrm{~K}\end{array}$ & $\begin{array}{l}1,6 \\
1,1\end{array}$ & $\begin{array}{l}4,25 \times 10^{-5} \\
1,63 \times 10^{-6}\end{array}$ & $\begin{array}{l}3,22 \\
5,00\end{array}$ \\
\hline D II & $\begin{array}{r}300 \mathrm{~K} \\
77 \mathrm{~K}\end{array}$ & $\begin{array}{l}1,60 \\
1,30\end{array}$ & $\begin{array}{l}2,18 \times 10^{-5} \\
1,06 \times 10^{-6}\end{array}$ & $\begin{array}{l}3,5 \\
4.97\end{array}$ \\
\hline
\end{tabular}

Si l'on regarde l'évolution des caractéristiques inverses courant-tension (Fig. 3) avec la température, par analogie avec les résultats sur les homojonctions, le courant s'apparente à un courant tunnel pour les cellules $\mathrm{N}$ et à un courant d'avalanche pour les cellules D I et D II. La loi de variation avec la température de $I_{\mathrm{Br}}$ et $k_{\mathrm{Br}}$ pour des températures supérieures à $160 \mathrm{~K}$ est donnée par les équations :

$$
\begin{aligned}
& \mathrm{N}: I\left(\mathrm{~A} / \mathrm{cm}^{2}\right)=1,07 \times 10^{-9} \exp \left(1,57 \times 10^{-2} \mathrm{~T}\right) \times \\
& \quad \times \exp (2,56 \mathrm{~V}) \\
& V_{\mathrm{Br}}(\mathrm{V})=2,75 \\
& \mathrm{D} \mathrm{I}: I\left(\mathrm{~A} / \mathrm{cm}^{2}\right)=4,60 \times 10^{-8} \times \\
& \quad \times \exp \left(2,24 \times 10^{-2} \mathrm{~T}\right) \\
& \quad \times \exp \left[6,64\left(1-1,8 \times 10^{-3} \mathrm{~T}\right) \mathrm{V}\right] \\
& V_{\mathrm{Br}}(\mathrm{V})=0,71\left(1+3,8 \times 10^{-3} \mathrm{~T}\right) .
\end{aligned}
$$

Pour des tensions inférieures à $V_{\mathrm{Br}}$, le modèle proposé par S. Martinuzzi [10] c'est-à-dire un cou- rant Zener assisté par des niveaux à l'interface, s'applique à nos caractéristiques.

2.2 CARACTÉRISTIQUES CAPACITÉ-TENSION. - La variation de l'inverse du carré de la capacité de la jonction avec la tension peut se représenter, en polarisation inverse, et au voisinage de zéro en polarisation directe, par une droite que nous caractérisons par sa pente et son intersection avec l'axe des tensions $V_{\infty}$ (Tableau IV). $C_{0}$ correspond à la capacité de la jonction pour $V=0$.

Tableau IV. - Résultats des courbes $1 / C^{2}=f(V)$ avant et après dégradation. $P$ représente la pente de la courbe.

$\begin{array}{lcccc} & & C_{0}\left(\mu \mathrm{F} / \mathrm{cm}^{2}\right) & P\left(\mu \mathrm{F}^{-2} \mathrm{~cm}^{4} \mathrm{~V}^{-1}\right) & V_{\infty}(\mathrm{V}) \\ & & - & - & - \\ \mathrm{N} & \mathrm{Av} & 0,55 & 1,22 & 2,70 \\ & \mathrm{Ap} & 0,31 & 1,5 & 6,80 \\ \mathrm{D} \mathrm{I} & \mathrm{Av} & 1,70 & 0,033 & 11,4 \\ & \mathrm{Ap} & 1,07 & 0,109 & 8,02 \\ & \mathrm{Av} & 1,20 & 0,14 & -6,2 \\ \mathrm{D} \text { II } & \mathrm{Ap} & 0,625 & 0,410 & 6,25\end{array}$

Avec la température l'allure des courbes n'est pas modifiée, lorsque la température diminue, $C_{0}$ diminue tandis que $V_{\infty}$ augmente (Fig. 4).

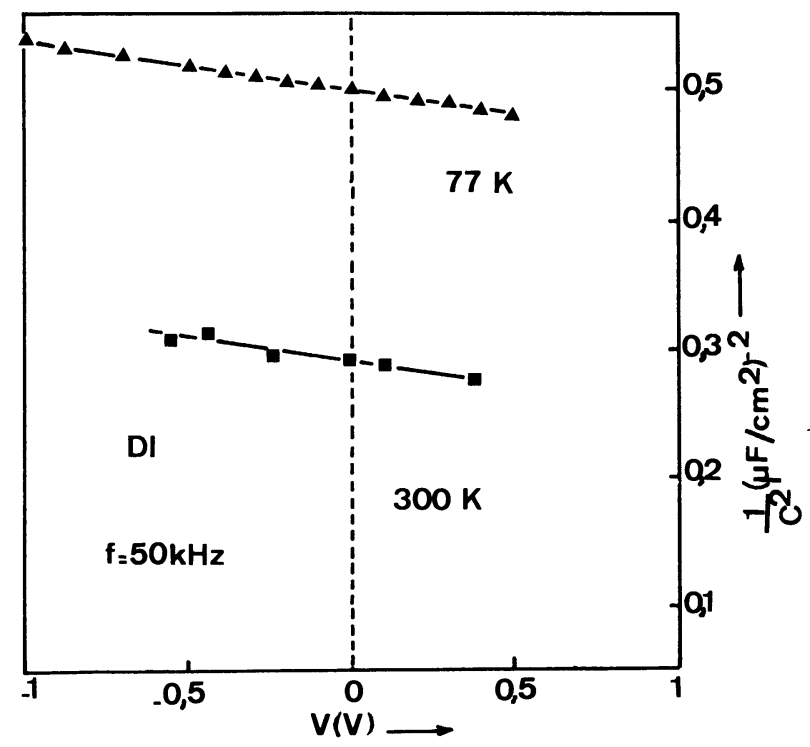

Fig. 4. - Variation de $1 / C^{2}$ en fonction de la tension appliquée à $300 \mathrm{~K}$ et à $77 \mathrm{~K}$.

2.3 EVOLUTION DES CARACTÉRISTIQUES ÉLECTRIQUES APRÈS UN TEST DE DÉGRADATION. - Certaines cellules ont subi un test de dégradation qui consiste à soumettre la cellule à un éclairement de $100 \mathrm{~mW} / \mathrm{cm}^{2}$ durant $100 \mathrm{~h}$ à une température de $100^{\circ} \mathrm{C}$. L'influence de ce traitement se traduit par une modification des caractéristiques courant-tension (Fig. 5). Sur les diodes dégradées, le courant inverse pour les faibles 


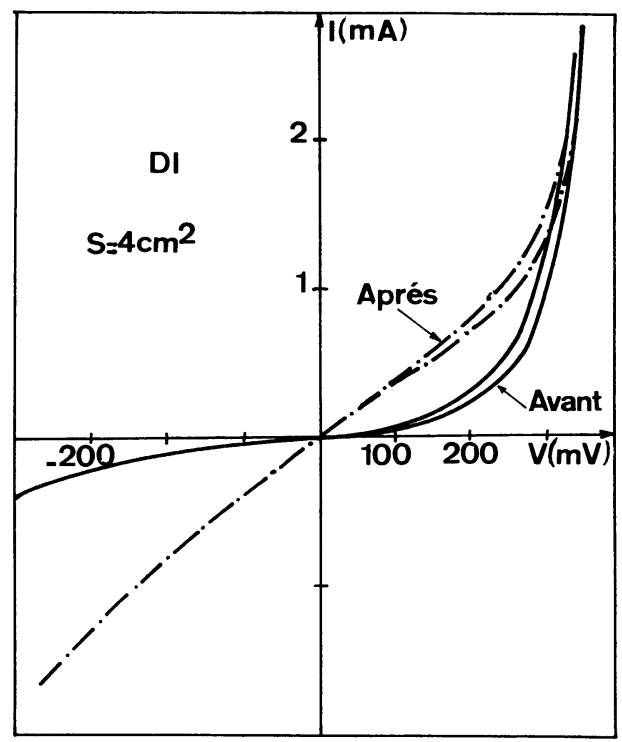

Fig. 5. - Evolution des caractéristiques $I-V$ après dégradation optique. Représentation métrique.

polarisations est supérieur en valeur absolue au courant direct (Fig. 6). Les modifications des paramètres $C_{0}, P$ et $V_{\infty}$ sont données sur le tableau IV.

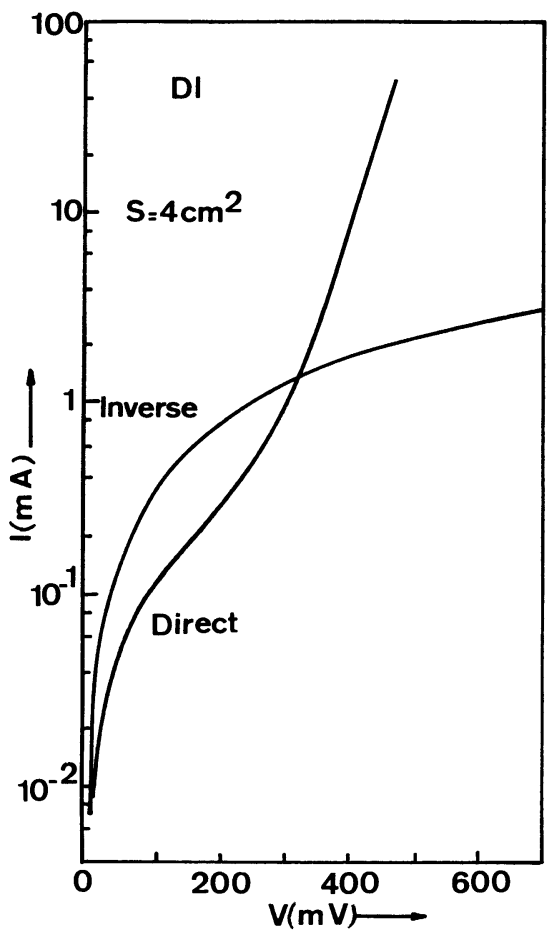

Fig. 6. - Modification des caractéristiques directes et inverses après dégradation optique. Représentation semi-logarithmique.

3. Discussion des résultats expérimentaux. - En accord avec les résultats de S. Martinuzzi [10] et J. Bernard [11] [12], les lois de courant à l'obscurité et sous éclairement correspondent à des mécanismes de conduction par effet tunnel comme le montre l'évolution de ces courants avec la température. La valeur anormalement élevée de la barrière $V_{\infty}$ déterminée par les mesures de capacité peut s'expliquer par l'existence d'une couche interfaciale entre le $\mathrm{CdS}$ et le $\mathrm{Cu}_{2} \mathrm{~S}$ avec la présence de charges aux limites de cette couche, comme nous allons le montrer.

3.1 MODIFICATION DE LA CAPACITÉ PAR UNE COUCHE INTERFACIALE [13, 14]. - Nous supposons qu'entre le $\mathrm{CdS}$ et le $\mathrm{Cu}_{2} \mathrm{~S}$ se trouve une couche d'épaisseur $\delta$ avec à chaque bord et à l'intérieur de celle-ci des charges $Q_{\mathrm{s} 1}$ et $Q_{\mathrm{s} 2}$ suivant la figure 7 .

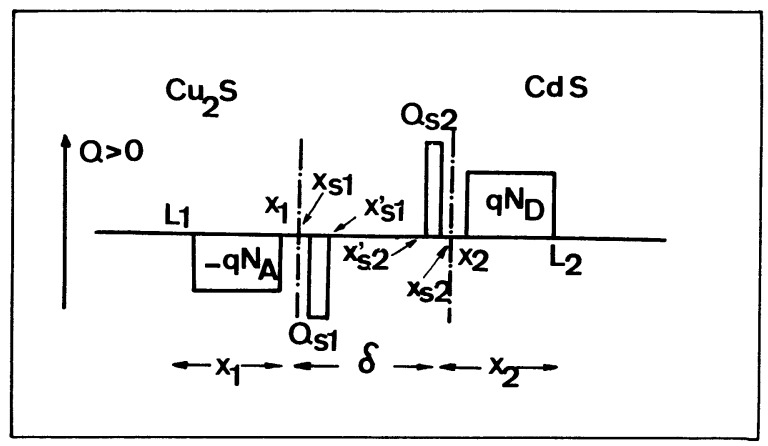

Fig. 7. - Schéma des répartitions des charges dans le cas d'une couche interfaciale entre le $\mathrm{Cu}_{2} \mathrm{~S}$ et le $\mathrm{CdS}$.

Les points $x_{1}, x_{\mathrm{s} 1}, x_{\mathrm{S} 1}^{\prime}$ sont confondus ainsi que $x_{2}$, $x_{\mathbf{S} 2}$ et $x_{\mathbf{S} 2}^{\prime}$; ils ne sont séparés sur le schéma que pour différencier les zones d'intégration. On appelle $V_{\mathrm{p}}$, $V_{\mathrm{S}}$ et $V_{\mathrm{n}}$ les chutes de tension dans le $\mathrm{Cu}_{2} \mathrm{~S}$, dans la couche interfaciale et dans le CdS; la somme est égale à $V_{\mathrm{b}}=V_{\mathrm{D}}-V$ où $V_{\mathrm{D}}$ est la tension de diffusion et $V$ la tension appliquée.

L'intégration de l'équation de Poisson entre $L_{1}$ et $x_{1}$ conduit à :

$$
\begin{aligned}
& \left(\frac{\mathrm{d} V}{\mathrm{~d} x}\right)_{x_{1}}=\frac{q N_{\mathrm{A}}}{\varepsilon_{1}}\left(x_{1}-L_{1}\right)=\frac{q N_{\mathrm{A}}}{\varepsilon_{1}} X_{1} \\
& V\left(x_{1}\right)=V_{L_{1}}+\frac{q N_{\mathrm{A}}}{2 \varepsilon_{1}}\left(x_{1}-L_{1}\right)^{2} .
\end{aligned}
$$

Entre $x_{1}$ et $x_{\mathbf{S} 1}^{\prime}$ nous avons :

$\varepsilon_{1}\left(\frac{\mathrm{d} V}{\mathrm{~d} x}\right)_{x_{1}}=\varepsilon_{\mathrm{S}}\left(\frac{\mathrm{d} V}{\mathrm{~d} x}\right)_{x_{\mathbf{S}}}$
$\varepsilon_{\mathrm{S}}\left(\frac{\mathrm{d} V}{\mathrm{~d} x}\right)_{x_{\mathbf{S} 1}}-\varepsilon_{\mathrm{S}}\left(\frac{\mathrm{d} V}{\mathrm{~d} x}\right)_{x_{\mathbf{S}} \mathbf{1}}=Q_{\mathrm{S} 1}$.

De la continuité du champ entre $x_{\mathbf{S} 1}^{\prime}$ et $x_{\mathbf{S} 2}^{\prime}$ et en supposant que le potentiel ne varie pas entre $x_{1}$ et $x_{\mathbf{S} 1}^{\prime}$ et entre $x_{\mathbf{S} 2}^{\prime}$ et $x_{\mathbf{2}}$ :

$$
\begin{aligned}
& \left(\frac{\mathrm{d} V}{\mathrm{~d} x}\right)_{x_{\mathbf{S} 2}}=\left(\frac{\mathrm{d} V}{\mathrm{~d} x}\right)_{x_{\mathbf{S}} \mathbf{}}=\frac{q N_{\mathrm{A}}}{\varepsilon_{\mathrm{S}}} X_{1}-\frac{Q_{\mathrm{S} 1}}{\varepsilon_{\mathrm{S}}} \\
& V\left(x_{2}\right)=V_{L_{1}}+\frac{q N_{\mathrm{A}}}{2 \varepsilon_{1}} X_{1}^{2}+\frac{q N_{\mathrm{A}}}{\varepsilon_{\mathrm{S}}} X_{1}-\frac{Q_{\mathrm{S} 1}}{\varepsilon_{\mathrm{S}}}
\end{aligned}
$$


Nous retrouvons entre $x_{\mathbf{s} 2}^{\prime}$ et $x_{2}$ les mêmes relations qu'entre $x_{\mathrm{s} 1}^{\prime}$ et $x_{1}$ :

$$
\begin{aligned}
& \left(\frac{\mathrm{d} V}{\mathrm{~d} x}\right)_{x_{\mathrm{S} 2}}-\left(\frac{\mathrm{d} V}{\mathrm{~d} x}\right)_{x_{\mathbf{S} 2}}=\frac{Q_{\mathrm{S} 2}}{\varepsilon_{\mathrm{S}}} \\
& \varepsilon_{2}\left(\frac{\mathrm{d} V}{\mathrm{~d} x}\right)_{x_{2}}=\varepsilon_{\mathrm{S}}\left(\frac{\mathrm{d} V}{\mathrm{~d} x}\right)_{x_{\mathbf{S} 2}}
\end{aligned}
$$

en intégrant l'équation de Poisson entre $x_{2}$ et $L_{2}$ on arrive pour le potentiel à :

$$
\begin{aligned}
V_{\mathrm{b}}= & V_{L_{2}}-V_{L_{1}}=\left[\frac{q N_{\mathrm{A}} X_{1}}{\varepsilon_{\mathrm{S}}}-\frac{Q_{\mathrm{S} 1}}{\varepsilon_{\mathrm{S}}}\right] \delta+ \\
& +\left[\frac{q N_{\mathrm{A}} X_{1}}{\varepsilon_{2}}-\frac{Q_{\mathrm{S} 1}}{\varepsilon_{2}}-\frac{Q_{\mathrm{S} 2}}{\varepsilon_{2}}\right] X_{2} \\
& +\frac{q N_{\mathrm{A}}}{2 \varepsilon_{1}} X_{1}^{2}-\frac{q N_{\mathrm{D}}}{2 \varepsilon_{2}} X_{2}^{2} .
\end{aligned}
$$$$
\text { (3) } \quad V_{\mathrm{n}}=\frac{q N_{\mathrm{D}}}{2 \varepsilon_{2}} X_{2}^{2} \text {. }
$$

En écrivant que le champ est nul en $L_{2}$ nous avons :

$q N_{\mathrm{A}} X_{1}-q N_{\mathrm{D}} X_{2}=Q_{\mathrm{S} 1}+Q_{\mathrm{S} 2}$

et l'équation (5) s'écrit :

En exprimant, à partir de la relation (8), $X_{1}$ en fonction de $X_{2}$, des équations précédentes on arrive à une équation du second degré (10) en $X_{2}$ dont on garde la solution positive (11)

$$
\begin{aligned}
\left(\frac{N_{\mathrm{A}}}{\varepsilon_{2}}\right. & \left.+\frac{N_{\mathrm{D}}}{\varepsilon_{1}}\right) X_{2}^{2}+2\left(\frac{N_{\mathrm{A}}}{\varepsilon_{\mathrm{S}}}+\frac{Q_{\mathrm{S} 1}+Q_{\mathrm{S} 2}}{q \varepsilon_{1}}\right) X_{2}+\frac{\left(Q_{\mathrm{S} 1}+Q_{\mathrm{S} 2}\right)^{2}}{\varepsilon_{1} q^{2} N_{\mathrm{D}}}+\frac{2 N_{\mathrm{A}} Q_{\mathrm{S} 2} \delta}{q N_{\mathrm{D}} \varepsilon_{\mathrm{S}}}-\frac{2 N_{\mathrm{A}} V_{\mathrm{b}}}{q N_{\mathrm{D}}}=0 \\
X_{2}= & \left\{\left[\left(\frac{N_{\mathrm{A}}}{\varepsilon_{\mathrm{S}}}+\frac{Q_{\mathrm{S} 1}+Q_{\mathrm{S} 2}}{q \varepsilon_{1}}\right)^{2}-\left(\frac{N_{\mathrm{A}}}{\varepsilon_{2}}+\frac{N_{\mathrm{D}}}{\varepsilon_{1}}\right)\left(\frac{\left(Q_{\mathrm{S} 1}+Q_{\mathrm{S} 2}\right)^{2}}{\varepsilon_{1} q^{2} N_{\mathrm{D}}}+\frac{2 N_{\mathrm{A}} Q_{\mathrm{S} 2} \delta}{q N_{\mathrm{D}} \varepsilon_{\mathrm{S}}}-\frac{2 N_{\mathrm{A}} V_{\mathrm{b}}}{q N_{\mathrm{D}}}\right)\right]^{1 / 2}-\right. \\
& \left.-\left(\frac{N_{\mathrm{A}}}{\varepsilon_{\mathrm{S}}}+\frac{Q_{\mathrm{S} 1}+Q_{\mathrm{S} 2}}{q \varepsilon_{1}}\right)\right\}\left(\frac{N_{\mathrm{A}}}{\varepsilon_{2}}+\frac{N_{\mathrm{D}}}{\varepsilon_{1}}\right)^{-1} .
\end{aligned}
$$

A ce stade nous faisons cinq hypothèses simplificatrices :

1. La variation de $Q_{\mathrm{S} 1}$ avec la tension est du même signe que celle de $q N_{\mathrm{A}} X_{1}$, et celle de $Q_{\mathrm{S} 2}$ du même signe que celle de $q N_{\mathrm{D}} X_{2}$.

2. $\mathrm{d}\left(Q_{\mathrm{s} 1}+Q_{\mathrm{s} 2}\right)=0$ et $Q_{\mathrm{s} 1}+Q_{\mathrm{s} 2}=0$.

3. $\varepsilon_{1}=\varepsilon_{2}=\varepsilon_{\mathrm{S}}=\varepsilon$.

4. $\delta$ est indépendant de la tension appliquée.

5. La concentration en accepteurs dans le $\mathrm{Cu}_{2} \mathrm{~S}$ est plus grande que celle des donneurs dans le CdS.

6. Nous supposons que la zone de charge d'espace se trouve dans le CdS.

Avec ces hypothèses (11) s'écrit :

$X_{2}=\left[\delta^{2}+\frac{2 \varepsilon V_{\mathrm{b}}}{q N_{\mathrm{D}}}-\frac{2 Q_{\mathrm{S} 2} \delta}{q N_{\mathrm{D}}}\right]^{1 / 2}-\delta$.

La capacité de la jonction s'écrit

$C=\left|\frac{\mathrm{d} Q}{\mathrm{~d} V}\right|$

où $Q=q N_{\mathrm{D}} X_{2}+Q_{\mathrm{s} 2}$ avec $Q_{\mathrm{s} 2}=Q_{\mathrm{s} 0}-C_{\mathrm{S}} V$.
Soit en tenant compte des hypothèses précédentes et des relations (3) à (11) :

$C=\frac{1-\frac{\delta}{\varepsilon} C_{\mathrm{S}}}{\left[\frac{\delta^{2}}{\varepsilon^{2}}+\left(\frac{2 V_{\mathrm{b}}}{q \varepsilon N_{\mathrm{D}}}-\frac{2 Q_{\mathrm{s} 2} \delta}{\varepsilon q N_{\mathrm{D}} \varepsilon}\right)\right]^{1 / 2}}+C_{\mathrm{S}}$

En utilisant d'une part le résultat théorique de l'expression de la capacité de la structure, en présence d'une couche interfaciale, et d'autre part le modèle d'effet tunnel par l'intermédiaire de pièges dans la bande interdite, il nous sera possible de comparer efficacement nos résultats expérimentaux aux valeurs calculées.

3.2 Conduction dans Le CAS DE L'existence D'UNE COUCHE INTERFACIALE. - Les caractéristiques expérimentales courant-tension peuvent être expliquées dans le cas d'une couche interfaciale suivant les différents mécanismes de conduction représentés sur la figure 8 . Nous supposons que le courant direct de la cellule se compose de deux courants $I_{1}$ et $I_{2}$ comme nous l'avons vérifié expérimentalement. Le courant $I_{2}$, le plus important, est dû à un courant 


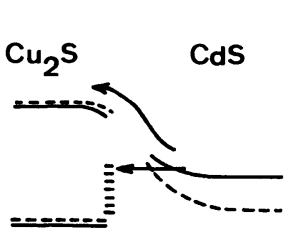

1

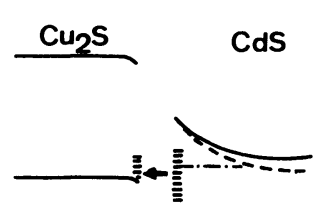

3 Direct
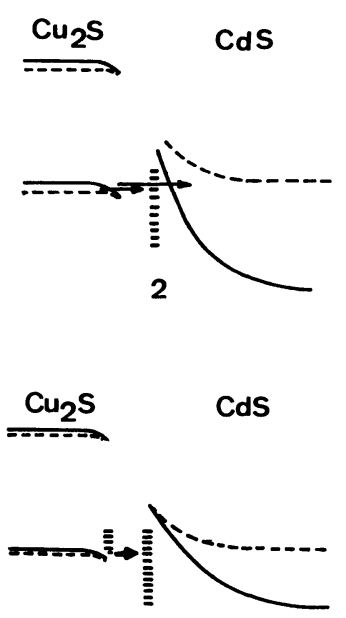

3 Inverse
Fig. 8. - 1. Pour les fortes polarisations directes, la conduction s'effectue par les électrons de la bande de conduction du CdS qui passent dans le $\mathrm{Cu}_{2} \mathrm{~S}$ par-dessus la barrière et par effet tunnel sur les pièges voisins du $\mathrm{Cu}_{2} \mathrm{~S}$. 2. Pour les fortes polarisations inverses,

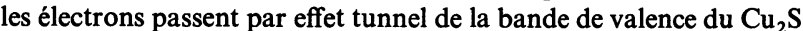
dans la bande de conduction du CdS et sur les pièges voisins du CdS. 3. Aux polarisations faibles, la conduction s'effectue principalement des défauts d'un côté de la couche interfaciale aux défauts du côté opposé. Ceci justifiant la même variation avec la température des courants direct et inverse.

tunnel, à travers la barrière $V_{\mathrm{b}}=V_{\mathrm{D}}-V$, de la bande de conduction du CdS vers la bande de valence du $\mathrm{Cu}_{2} \mathrm{~S}$ via les pièges à l'interface. Le courant le plus faible $I_{1}$ est régi par le passage par effet tunnel de pièges à pièges, à travers la couche interfaciale, des électrons se trouvant aux limites de celle-ci. Ces deux courants sont du même type que celui proposé par Riben et Feucht [15], [16] et S. Martinuzzi [10] et correspondent à un effet tunnel par les niveaux d'interfaces.

La probabilité de passage des électrons dans les deux cas s'écrit :

$$
T=\exp -\frac{4}{3} \frac{\sqrt{2 m}}{q \hbar} \frac{E_{\mathrm{\imath}}^{3 / 2}}{\varepsilon}
$$

où $E$ est la hauteur de la barrière que franchit l'électron et $\delta$ le champ électrique. L'expression des courant donne

$I_{1} \sim \exp -\frac{4}{3} \frac{\sqrt{2 q m}}{\hbar} V_{\mathrm{s}}^{1 / 2} \delta$

pour le premier courant, et

$I_{2} \backsim \exp -\frac{4}{3} \frac{\sqrt{2 q m}}{\hbar} \frac{V_{\mathrm{b}}^{3 / 2}}{q N_{\mathrm{D}} X_{2} / \varepsilon}$

pour le deuxième courant.

Dans les expressions ci-dessus $V_{\mathrm{S}}, V_{\mathrm{b}}, X_{2}$ dépendent de $V$ ainsi que l'expression de la capacité $C$ (13).
Nos résultats expérimentaux ont montré qu'en première approximation $1 / C^{2}$ et $\operatorname{Ln} I$ étaient des fonctions linéaires du potentiel $V$. Aussi nous est-il possible de choisir arbitrairement des valeurs de $N_{\mathrm{D}}$, $\delta, Q_{\mathrm{s} 0}, C_{\mathrm{s}}$ et $V_{\mathrm{D}}$ conduisant à des valeurs de la pente $P$ de la droite $1 / C^{2}=f(V)$, de son intersection $V_{\infty}$ avec l'axe des $V$ et des coefficients $a_{1}$ et $a_{2}$ rentrant dans l'expression des courants $I_{1}$ et $I_{2}$, compatibles avec celles déduites des expériences.

Le tableau $\mathrm{V}$ donne un tel choix pour 2 types de cellules, l'une type N, l'autre type D I. Nous remarquons que la concordance est bonne sauf pour la diode $\mathrm{D} I$ dans le cas du coefficient $a_{1}$. Ces résultats justifient le modèle de conduction proposé pour les cellules évaporées $\mathrm{Cu}_{2} \mathrm{~S}-\mathrm{CdS}$ (S.A.T.).

Tableau V.

\begin{tabular}{|c|c|c|c|}
\hline $\begin{array}{c}C_{0} \\
\left(\mu \mathrm{F} / \mathrm{cm}^{2}\right)\end{array}$ & $\begin{array}{l}\text { expérim. } \\
\text { calculé }\end{array}$ & $\begin{array}{l}0,55 \\
0,52\end{array}$ & Cellule type $\mathrm{N}$ \\
\hline $\begin{array}{c}P \\
\left(\mu \mathrm{F}^{-2} \mathrm{~cm}^{4} \mathrm{~V}^{-1}\right)\end{array}$ & $\begin{array}{l}\text { expérim. } \\
\text { calculé }\end{array}$ & $\begin{array}{l}1,22 \\
1,32\end{array}$ & $\begin{array}{c}\text { avec } \\
N_{\mathrm{D}}=10^{18} \mathrm{~cm}^{-3}\end{array}$ \\
\hline$V_{\infty}(\mathrm{V})$ & $\begin{array}{l}\text { expérim. } \\
\text { calculé }\end{array}$ & $\begin{array}{l}2,70 \\
2,79\end{array}$ & $\delta=70 \AA$ \\
\hline$a^{-1}\left(\mathrm{~V}^{-1}\right)$ & $\begin{array}{l}\text { expérim. } \\
\text { calculé }\end{array}$ & $\begin{array}{l}11 \\
11,2\end{array}$ & $\begin{aligned} Q_{\mathrm{s} 0} & =1,108 \times 10^{-3} \mathrm{Cb} / \mathrm{m}^{2} \\
V_{\mathrm{D}} & =0,6 \mathrm{~V}\end{aligned}$ \\
\hline$a^{-2}\left(\mathrm{~V}^{-1}\right)$ & $\begin{array}{l}\text { expérim. } \\
\text { calculé }\end{array}$ & $\begin{array}{l}35 \\
35,1\end{array}$ & $C_{\mathrm{s}}=0,279 \mu \mathrm{F} / \mathrm{cm}^{2}$ \\
\hline $\begin{array}{c}C_{0} \\
\left(\mu \mathrm{F} / \mathrm{cm}^{2}\right)\end{array}$ & $\begin{array}{l}\text { expérim. } \\
\text { calculé }\end{array}$ & $\begin{array}{l}1,7 \\
1,66\end{array}$ & $\begin{array}{c}\text { Cellule type D I } \\
\text { avec }\end{array}$ \\
\hline $\begin{array}{c}P \\
\left.\mu \mathrm{F}^{-2} \mathrm{~cm}^{4} \mathrm{~V}^{-1}\right)\end{array}$ & $\begin{array}{l}\text { expérim. } \\
\text { calculé }\end{array}$ & $\begin{array}{l}0,033 \\
0,032\end{array}$ & $N_{\mathrm{D}}=8 \times 10^{18} \mathrm{~cm}^{-3}$ \\
\hline$V_{\infty}(\mathrm{V})$ & $\begin{array}{l}\text { expérim. } \\
\text { calculé }\end{array}$ & $\begin{array}{l}11,4 \\
11,3\end{array}$ & $\begin{aligned} \delta & =35 \AA \\
Q_{\mathrm{S} 0} & =4,7 \times 10^{-3} \mathrm{Cb} / \mathrm{m}^{2}\end{aligned}$ \\
\hline$a_{1}\left(\mathrm{~V}^{-1}\right)$ & $\begin{array}{l}\text { expérim. } \\
\text { calculé }\end{array}$ & $\begin{array}{l}9 \\
6,3\end{array}$ & $V_{\mathrm{D}}=0,8 \mathrm{~V}$ \\
\hline$a_{2}\left(\mathrm{~V}^{-1}\right)$ & $\begin{array}{l}\text { expérim. } \\
\text { calculé }\end{array}$ & $\begin{array}{l}30 \\
30,9\end{array}$ & $C_{\mathrm{s}}=1,3 \mu \mathrm{F} / \mathrm{cm}^{2}$ \\
\hline
\end{tabular}

4. Conclusion. - Le sulfure de cuivre, qui constitue la couche $p$ de la cellule photovoltaïque, a une concentration très voisine de la stochiométrie. Nos mesures cristallographiques ont montré que la transformation de la chalcocite $\gamma$ en chalcocite $\beta$ avait lieu aux mêmes températures que sur les échantillons massifs. L'évolution du courant de court-circuit varie comme l'intensité des raies de la chalcocite (Fig. 9). Si on se réfère aux résultats obtenus sur des échantillons massifs la concentration en accepteurs dans le $\mathrm{Cu}_{2} \mathrm{~S}$ en présence de cadmium doit être comprise entre $10^{18}$ et $10^{19} \mathrm{~cm}^{-3}$.

Les cellules de la S.A.T. sont réalisées à partir d'un sulfure de cadmium fortement dopé. Les lois des courants à l'obscurité et sous éclairement correspondent à des mécanismes de conduction par effet tunnel comme le montre l'évolution de ces courants avec la température. La variation du courant de saturation $\left(I_{\mathrm{S}}=I_{\mathrm{SO}} \exp c T\right)$ est à comparer à celle observée par Riben et Feucht [15, 16], sur des cellules $\mathrm{Ga}-\mathrm{GaAs}$ et par Martinuzzi [10] sur des cellules $\mathrm{Cu}_{2} \mathrm{~S}-\mathrm{CdS}$. L'hypothèse d'une couche interfaciale 
a)

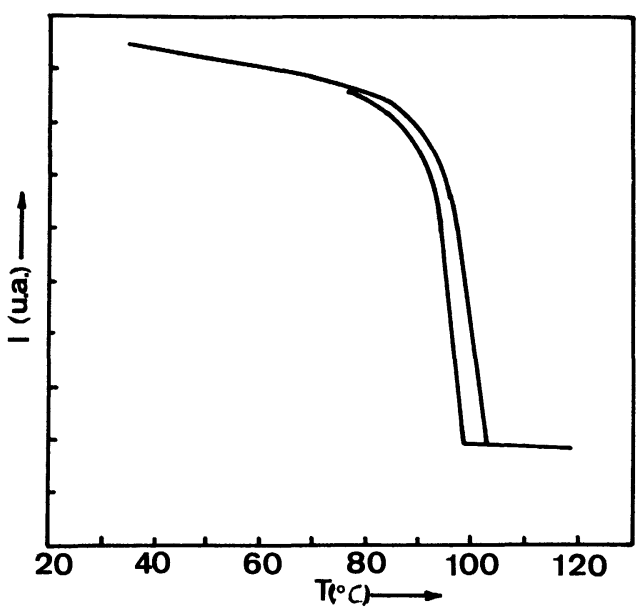

b)

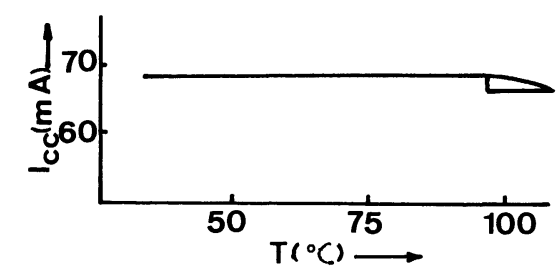

Fig. 9. - a) Variation de l'intensité d'une raie de diffraction $\mathrm{X}$ de la chalcocite $\gamma . b$ ) Variation du courant de court-circuit $I_{\text {cc }}$ avec la température. limitée par des états d'interfaces entre le $\mathrm{Cu}_{2} \mathrm{~S}$ et le $\mathrm{CdS}$, permet d'expliquer d'une part, les allures des caractéristiques $I-V$ et d'autre part, les allures des courbes de l'inverse du carré de la capacité de la cellule avec la tension appliquée. Dans ce modèle la conduction aux faibles polarisations correspondrait à des transitions tunnels d'états localisés à états localisés de chaque côté de la couche interfaciale, ce qụi expliquerait, par un phénomène d'épuisement, la saturation qu'on observe sur le courant direct pour des cellules dégradées. Aux fortes polarisations (directes et inverses) on aurait un phénomène de conduction par effet tunnel de la bande de conduction vers les états d'interfaces ou pour les fortes polarisations inverses par effet tunnel de bande à bande [17].

Remerciements. - Nous tenons à remercier Monsieur Nguyen Duy Thuoc de la Société Anonyme des Télécommunications; qu'il trouve ici l'expression de nos plus vifs remerciements pour avoir fourni les cellules et pour les nombreuses et fructueuses discussions que nous avons eues tout au long de cette étude. Ce travail a été supporté financièrement par le Centre National d'Etudes Spatiales.

\section{Bibliographie}

[1] Bernard, J., Techn. Note NT 02.2 CNES, Toulouse (1967).

[2] Palz, W., Cohen-Solal, G., Vedel, J., Fremy, J., Duy, T. N., VALERIO, J., 7th Photovolt. Spec. Conf. Rec. (1968) (IEEE) (1968) p. 54.

[3] Palz, W., Besson, J., Duy, T. N., Vedel, J., 10th Photovolt. Spec. Conf. Rec. (1973) (IEEE) (1974) p. 69.

[4] Palz, W., Besson, J., Fremy, J., DuY, T. N., Vedel, J., 8th Photovolt. Spec. Conf. Rec. (1970) (IEEE) (1970) p. 16.

[5] Coste, G., Fremy, J., DuY, T. N., Proc. Int. Colloq. Solar Cells (1971) p. 187.

[6] Palz, W., Besson, J., Duy, T. N., Vedel, J., 9th Photovolt. Spec. Conf. Rec. (1973) (IEEE) p. 91

[7] LuQuet, H., Thèse de Spécialité, Montpellier (1969).

[8] LuQuet, H., Szepessy, L., Bougnot, J., Savelli, M., 11th Photovolt. Spec. Conf. Rec. (1975) (IEEE) p. 445.

[9] Luquet, H., Moussalli, G., Bougnot, J., Savelli, M.,
Comples International Meeting " Heliotechnique and Development », Dharhan (Arabie Saoudite), 2-6 nov. 1975 1 (1976) 617-623.

[10] Martinuzzi, S., Mallen, O., Phys. Status Solidi (a) 16 (1973) 339.

[11] Bernard, J., Amand, T., Int. Conf. on Solar Electricity, Toulouse, France (1976) p. 309.

[12] Bernard, J., Vormus, J. P., Photovoltaic Solar Energy Conf., Luxembourg (1977) p. 570.

[13] Goodman, A. M., J. Appl. Phys. 34 (1963) 329.

[14] Goodman, A. M., Bull. Amer. Phys. Soc. 8 (1963) 210.

[15] Riben, A. R., Feucht, D. L., Solid Status Electron. 9 (1966) 1055.

[16] Riben, A. R., Feucht, D. L., Int. J. Electron. 20-6 (1966) 583.

[17] LuQueT, H., Thèse de Doctorat d'Etat, Montpellier (1977). 